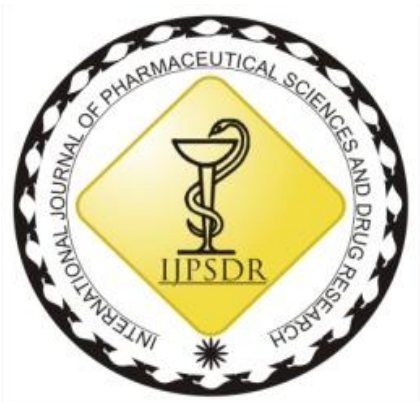

ISSN: 0975-248X

RESEARCH ARTICLE CODEN (USA): IJPSPP $($ (c) $)$ EY-NC-SA

\title{
Pure and SrBa Dual Doped ZnO Nanoparticles Prepared by Co- precipitation Method and Their Structural, Optical and Antimicrobial Properties
}

\author{
M. Karthikeyan ${ }^{1}$, A. Jafar Ahamed ${ }^{2}$, P. Vijaya Kumar ${ }^{3}$ \\ ${ }^{1}$ Department of Chemistry, Periyar Maniammai Institute of Science and Technology, Vallam, Thanjavur-613403, \\ Tamil Nadu, India \\ ${ }^{2}$ Post Graduate and Research Department of Chemistry, Jamal Mohamed College (Autonomous), Affiliated to \\ Bharathidasan University, Tiruchirappalli-620020, Tamil Nadu, India \\ ${ }^{3}$ Department of Chemistry, Jairams Arts and Science College, Affiliated to Bharathidasan University, Karur-639003, \\ Tamil Nadu, India
}

Copyright (C) 2019 M. Karthikeyan et al. This is an open access article distributed under the terms of the Creative Commons AttributionNonCommercial-ShareAlike 4.0 International License which allows others to remix, tweak, and build upon the work non-commercially, as long as the author is credited and the new creations are licensed under the identical terms.

\begin{abstract}
The present investigation, the successful preparation of pure ZnO (Z1) NPs and SrBa dual doped ZnO (Z2) NPs by chemical co-precipitation technique without use of any capping agent. The structural and morphological properties of Z1 and Z2 NPs were analyzed using X-ray diffraction (XRD) studies, Field emission scanning electron microscopy (FESEM), Elemental analysis (EDAX), Fourier transform infrared spectroscopy (FTIR). An optical property was studied by UV-Vis spectroscopy and Photoluminescence (PL) spectra. The antimicrobial activity of Z1 and Z2 NPs has been investigated against Staphylococcus aureus and Klebsiella pneumoniae bacterial strains. It has been interestingly observed that Z2 NPs has enhanced the inhibitory activity than that of Z1 NPs against $S$. aureus and more efficiently than the K. pneumoniae bacterial strain.
\end{abstract}

Keywords: Zinc Oxide, Nanoparticles, XRD, S. aureus and Antimicrobial activity.

DOI: 10.25004/IJPSDR.2019.110602

Int. J. Pharm. Sci. Drug Res. 2019; 11(6): 284-288

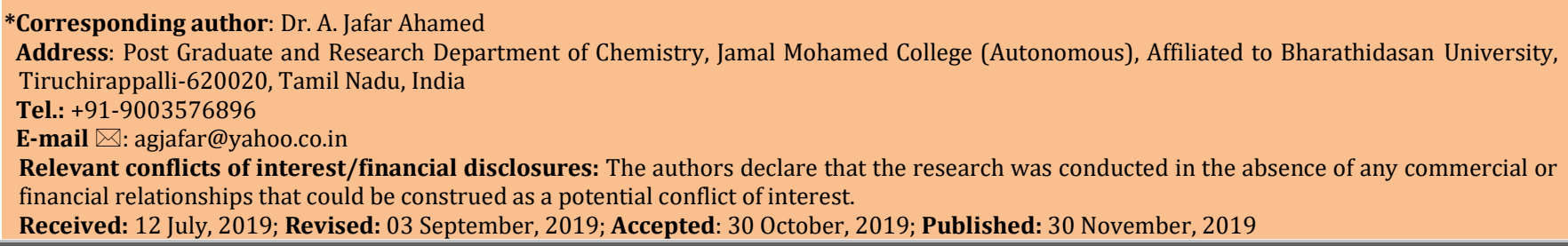

\section{INTRODUCTION}

Nowadays, one-dimensional (1D) nanostructures like nanowires, nanobelts, nanorods, and nanotubes have paying attention a great deal of interest in academic as well as industrial research. [1-2] These nanostructures find applications in an electronic devices and circuits, optoelectronic integrated circuits, electrochemical and electromechanical devices, etc. [3-4]

$\mathrm{ZnO}$ is an important n-type II-VI semiconductor NPs having direct band gap $3.37 \mathrm{eV}$, in addition with large excitation energy of $60 \mathrm{meV}$. Due to this property it has eminent uses such as luminescence, transparent 
electronics, optoelectronics, ultraviolet (UV) light emitters, piezoelectric devices etc. [5-6] $\mathrm{ZnO}$ is also suitable for UV screening applications, because it has low toxicity, high chemical stability and it is effective to inhibit pathogenic organisms. [7] In previous studies, $\mathrm{ZnO}$ reveals the room temperature ferromagnetism (RTFM), when the metallic dopants like $\mathrm{Al}, \mathrm{Co}, \mathrm{Mg}$ and $\mathrm{Mn}$ etc., are successfully doped in $\mathrm{ZnO}$. [8] Earlier reports, pure and dual doped (Co, Ag) $\mathrm{ZnO}$ NPs were manufactured by co-precipitation method without use of capping agent. [9] In the present investigation, we have planned to prepare pure and $\mathrm{SrBa}$ dual doped ZnO NPs by chemical co-precipitation method and their structural, optical and antimicrobial properties have been studied.

\section{MATERIALS AND METHODS}

Preparation of pure $\mathrm{ZnO}(\mathrm{Z1})$ and SrBa dual doped ZnO NPs (Z2)

To prepare $0.1 \mathrm{M} \mathrm{Zn}\left(\mathrm{NO}_{3}\right)_{2} \cdot 6 \mathrm{H}_{2} \mathrm{O}$ solution by using double distilled water under constant stirring. While at room temperature, solution of $0.8 \mathrm{M} \mathrm{NaOH}$ was added drop by drop. It was stirred constantly at a temperature of $80 \pm 5^{\circ} \mathrm{C}$ for 4 hours. Afterward end of the reaction, it was allowed to settle for overnight and the supernatant liquid was discarded. The obtained white coloured precipitate was thoroughly washed with double distilled water to remove all the other ions and then centrifuged at $3000 \mathrm{rpm}$ for 10 minutes. The final precipitate was dried in a hot air oven at $80^{\circ} \mathrm{C}$ for 2 hours. The above resultant dried powders was crushed and calcined at $700^{\circ} \mathrm{C}$ for 5 hours. The final product $\mathrm{ZnO}(\mathrm{Z1})$ was stored in air tight container for further analysis.

Similarly, in the preparation of $\mathrm{SrBa}$ dual doped $\mathrm{ZnO}$ $\mathrm{NPs}$, the required amount of $0.097 \mathrm{M} \mathrm{Zn}\left(\mathrm{NO}_{3}\right)_{2} \cdot 6 \mathrm{H}_{2} \mathrm{O}$, $0.002 \mathrm{M} \mathrm{Sr}\left(\mathrm{NO}_{3}\right)_{2} .6 \mathrm{H}_{2} \mathrm{O}, 0.001 \mathrm{M} \mathrm{Ba}\left(\mathrm{NO}_{3}\right)_{2} .6 \mathrm{H}_{2} \mathrm{O}$ was completely dissolved in deionized water, aqueous $\mathrm{NaOH}(0.8 \mathrm{M})$ solution was added drop wise to the above aqueous solution and stirred at $80 \pm 5^{\circ} \mathrm{C}$ for 4 hours. The white colour precipitate was formed slowly, which was washed several times with double distilled water and ethanol. The precipitate was dried at $120^{\circ} \mathrm{C}$. Thus, SrBa dual doped $\mathrm{ZnO}$ (Z2) nanopowder was obtained. This sample was annealed at $700^{\circ} \mathrm{C}$ for 5 hours.

\section{Antimicrobial Assay}

Antimicrobial activity of the Z1 and Z2 NPs was carried out by agar well diffusion method against two pathogenic bacterial strains namely $S$. aureus (gram positive) and K. pneumoniae (gram negative) on MullerHinton agar, according to the guidelines of Clinical and Laboratory Standards Institute (CLSI). [10] The media plates (MHA) were streaked with bacteria for 2-3 times by rotating the plate at $60^{\circ}$ angle for each streak to ensure homogeneous distribution of the inoculums. Then the agar plates were swabbed with $100 \mathrm{ml}$ each of overnight cultures of $S$. aureus and K. pneumoniae using a sterile L-shaped glass rod. Using a sterile cork-borer, wells $(6 \mathrm{~mm})$ were created in each petri plate. The concentration of Z1 and Z2 NPs $1.5 \mu \mathrm{g} / \mathrm{ml}$ for both gram positive and gram negative bacteria were loaded onto the petri plates followed by incubation for $24 \mathrm{~h}$ at $37^{\circ} \mathrm{C}$ for bacteria. After the incubation period, the diameter of the zone of inhibition (DZI) was recorded. Amoxicillin (Hi-Media) was used as the positive control against gram negative and gram positive bacteria to compare the efficacy of the test samples. We have simultaneously studied whether the dimethyl sulfoxide (DMSO) without the Z1 and Z2 NPs plays any active role as a biocide or not. As there is no any disc zone of inhibition observed, it confirms that DMSO doesn't show any biocidal property.

\section{Characterization Studies}

The Z1 and Z2 NPs were characterized by X-ray diffractometer (model: X'PERT PRO PAN analytical). The diffraction pattern was recorded in the range of $25^{\circ}-80^{\circ}$ for the Z1 and Z2 NPs, at the monochromatic wavelength of $1.54 \AA$. The morphological features of the sample were measured by Field emission scanning electron microscopy (Model: Carl Zess 55). The Z1 and Z2 NPs were analyzed by EDAX (model: ULTRA 55). The FT-IR spectrum was recorded in the range of 400$4000 \mathrm{~cm}^{-1}$ by using Perkin-Elmer spectrometer. Ultraviolet-visible (UV-Vis) spectra of Z1 and Z2 NPs were recorded on a Perkin-Elmer UV-Lambda 25 spectrophotometer (Perkin-Elmer, Norwalk, Connecticut). The PL emission study of the sample was carried out using Horiba Jobin YVON spectrofluorometer (model: FLUOROMAX-4, $450 \mathrm{~W}$ high pressure xenon lamp as the excitation source, photomultiplier at a range $325-550 \mathrm{~nm}$ ).

\section{RESULTS AND DISCUSSION}

\section{$X$-ray diffraction studies}

The XRD pattern of Z1 and Z2 NPs are shown in Fig. 1. The XRD peak position of at angles $(2 \theta)$ of 31.732, 34.320 and 36.354 , consistent to the (100), (002) and (101) planes of the $\mathrm{ZnO}$ NPs, respectively. Correspondingly, another peak position appeared at angles $(2 \theta)$ of $47.43,56.49,62.57,66.27,68.12,69.04$ and 76.99 related to the (102), (110), (103), (112), (201), (004) and (202) planes of the ZnO NPs, respectively. The standard X-ray diffraction peaks show that the hexagonal wurtzite structure of ZnO NPs with space group of p63mc and also confirmed by the JCPDS data (card No: 361451). This results are also witnessed that there is no impurity phase found in the $\mathrm{Sr}^{2+}$ and $\mathrm{Ba}^{2+}$ dual doped ZnO NPs, because of ionic radii of Sr (1.12 $\AA)$ and $\mathrm{Ba}(1.35 \AA)$. The average crystallite size of the samples are calculated by Debye Scherrer's relation,

Average crystallite size (D) $=\frac{0.9 \lambda}{\beta \cos \theta} \quad$--- (1)

Where $D$ is the crystallite size, $\lambda$ is the wavelength (1.5406 $\AA \mathrm{CuKa}), \theta$ is the Bragg diffraction angle and $\beta$ is the full width at half maximum (FWHM). The average crystallite sizes of Z1 and Z2 NPs were calculated to be $42 \mathrm{~nm}$ and $35 \mathrm{~nm}$. Because of SrBa dual 
doping in $\mathrm{ZnO}$ nanostructures, the particles size of its results decreases. It is due to distortion in the $\mathrm{ZnO}$ matrix by $\mathrm{Sr}^{2+}, \mathrm{Ba}^{2+}$ dopant ions, which increases the rate of growth of $\mathrm{ZnO}$.

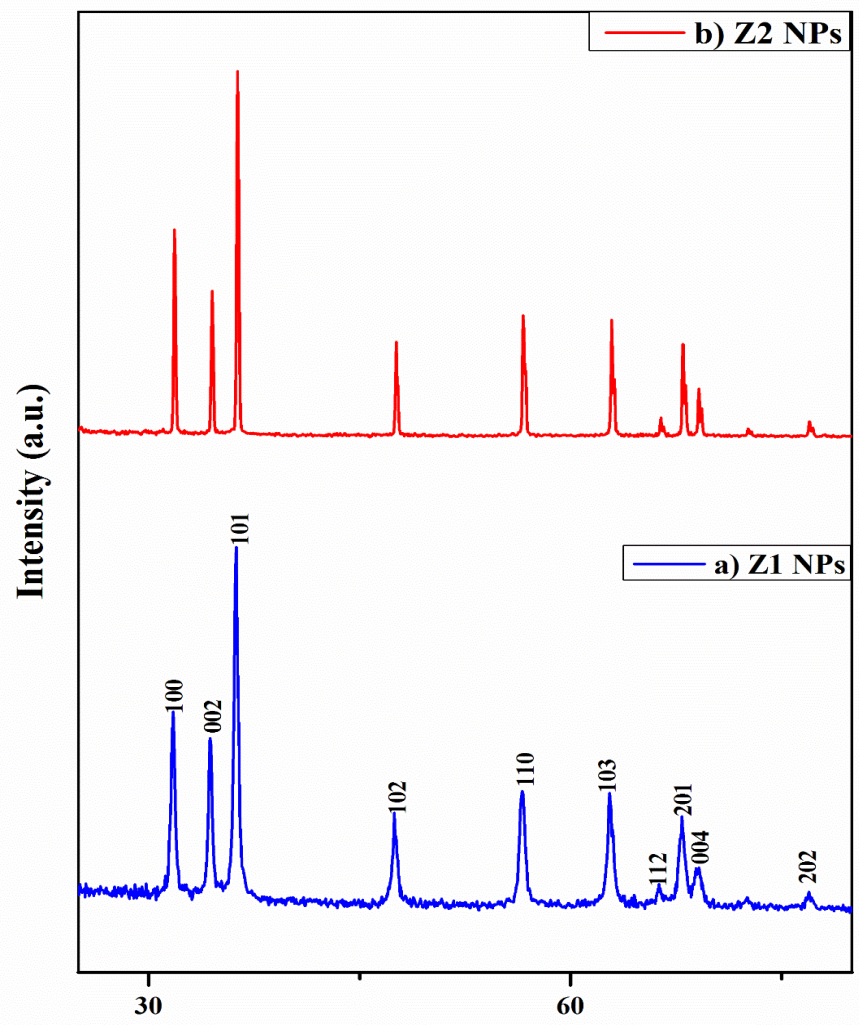

2 Theta Degree

Fig. 1: XRD pattern of a) Z1 NPs b) Z2 NPs
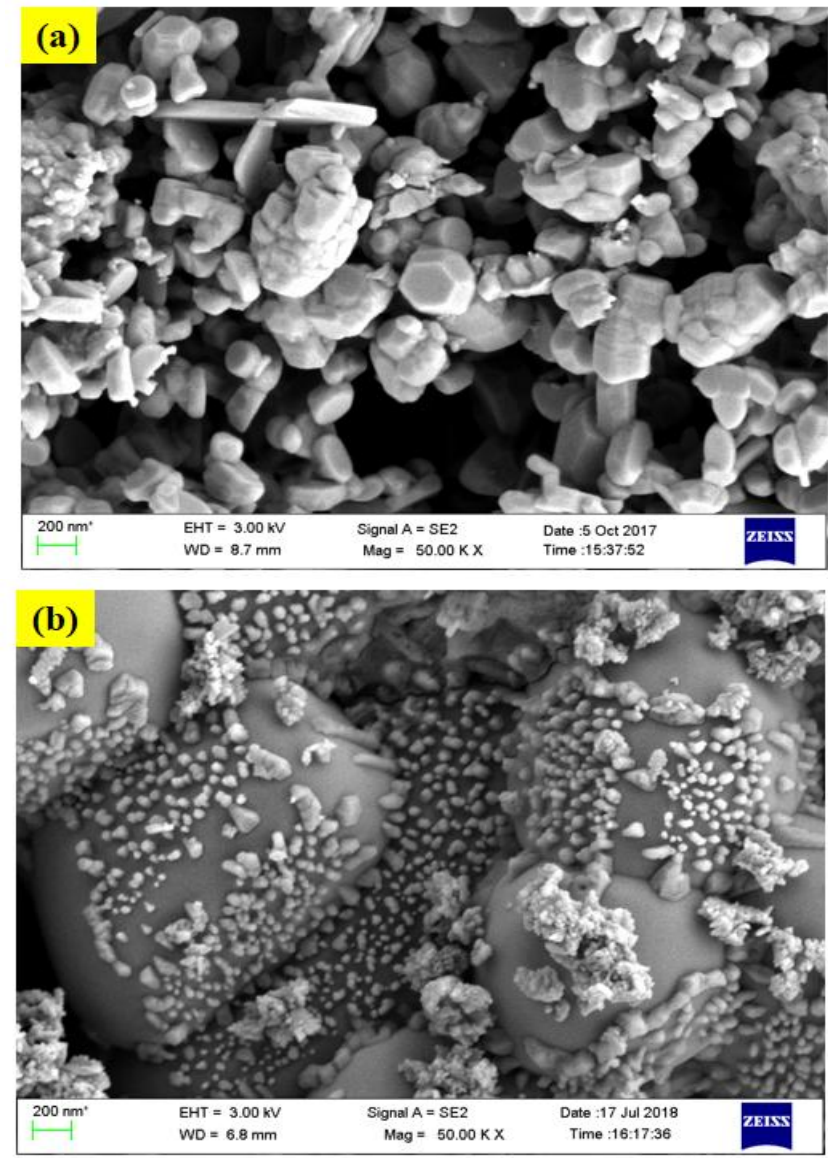

Fig. 2: FESEM images of a) Z1 NPs b) Z2 NPs
Morphological analysis

The surface morphology of the Z1 and Z2 NPs are shown in Fig. 2. From the FESEM images, the spherical and hexagonal like nanostructures were observed in Z1 NPs and Z2 NPs respectively. The average particle sizes were observed at $41 \mathrm{~nm}$ and $37 \mathrm{~nm}$ for Z1 and Z2 NPs respectively. The thicknesses of the nanoparticles are reduced, because the alkaline metal ions decrease the nucleation and subsequent growth rate of $\mathrm{ZnO} N P$ s. Elemental analysis

The EDAX spectra used to analyse the elemental composition of Z1 and Z2 NPs and it is shown in Fig. 3. In the $\mathrm{Z} 2$ sample, $\mathrm{Sr}$ and Ba composition is observed at $2.84 \%$ and $1.72 \%$ and the $\mathrm{Zn}$ and $\mathrm{O}$ are $67.39 \%$ and $21.15 \%$ respectively. In the $Z 1$ sample the chemical composition of $\mathrm{Zn}$ and $\mathrm{O}$ are found as $83.70 \%$ and $16.30 \%$ respectively. The EDAX spectrum has indicated the existence of $\mathrm{Zn}, \mathrm{O}, \mathrm{Sr}$, and $\mathrm{Ba}$ for the synthesized NPs.
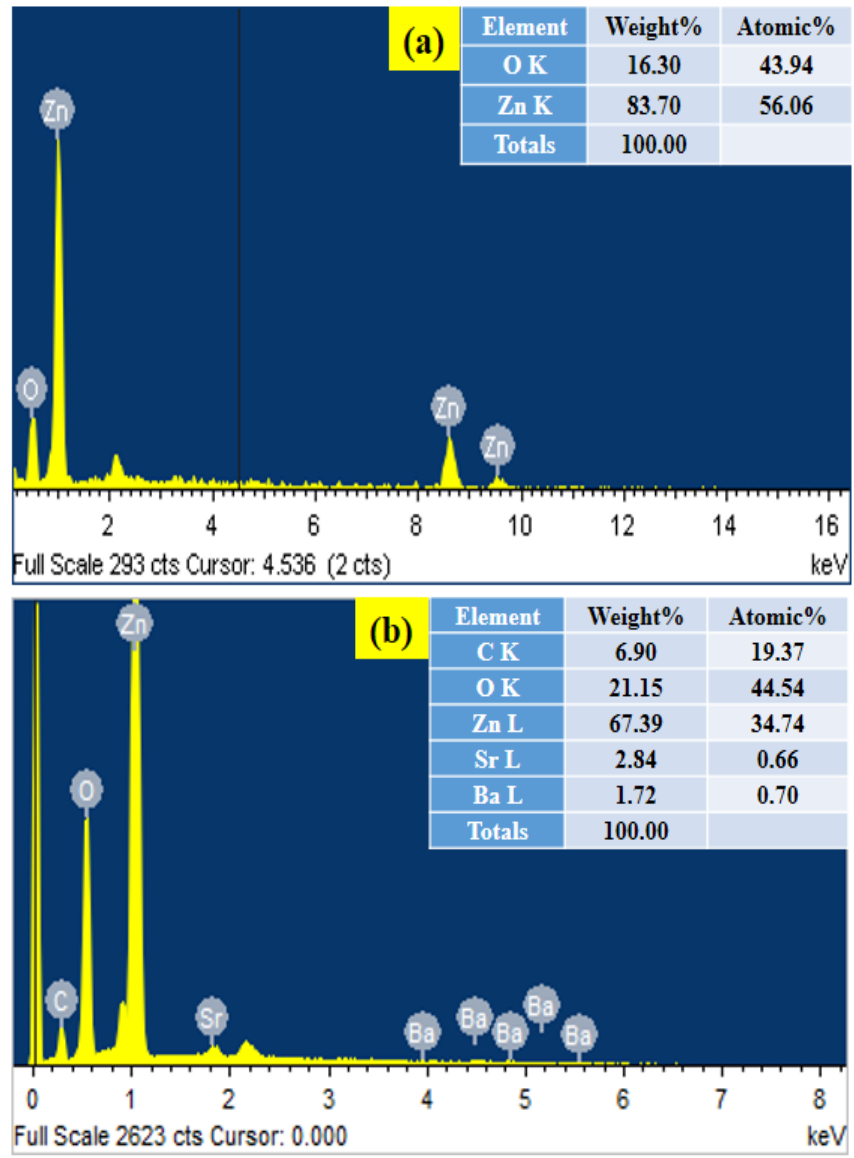

Fig. 3: EDAX spectra of a) Z1 NPs b) Z2 NPs

\section{Functional group analysis}

Fig. 4 shows that the FT-IR spectrum of the Z1 and Z2 NPs. The prepared Z1 and Z2 NPs were analyzed by FT-IR spectroscopy in the range from 400 to $4000 \mathrm{~cm}^{-1}$ at room temperature. It comprises several characteristic bands. The vibrational stretching frequency mode of $\mathrm{O}$ $\mathrm{H}$ band observed at a peak position in the range of $3020-3650 \mathrm{~cm}^{-1}$. [11] The peak in the range of $445 \mathrm{~cm}^{-1}$ corresponds to Zn-O stretching for Z1 NPs and in the case of Z2 NPs Zn-O stretching found to be $490 \mathrm{~cm}^{-1}$. 
Due to $\mathrm{H}-\mathrm{O}-\mathrm{H}$ bending vibration peak is observed at $1635 \mathrm{~cm}^{-1}$ which is assigned to a small amount of $\mathrm{H}_{2} \mathrm{O}$ in Z1 NPs. [12] The asymmetric C-O stretching bands to be $1112 \mathrm{~cm}^{-1}$ and asymmetric C-H bands are observed at $2924 \mathrm{~cm}^{-1}$ for Z1 and Z2 NPs.

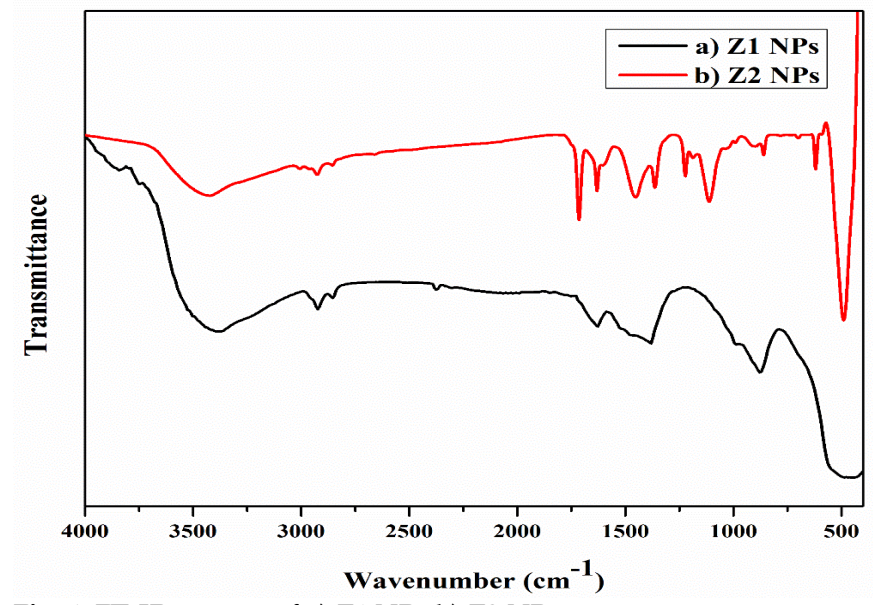

Fig. 4: FT-IR spectra of a) Z1 NPs b) Z2 NPs

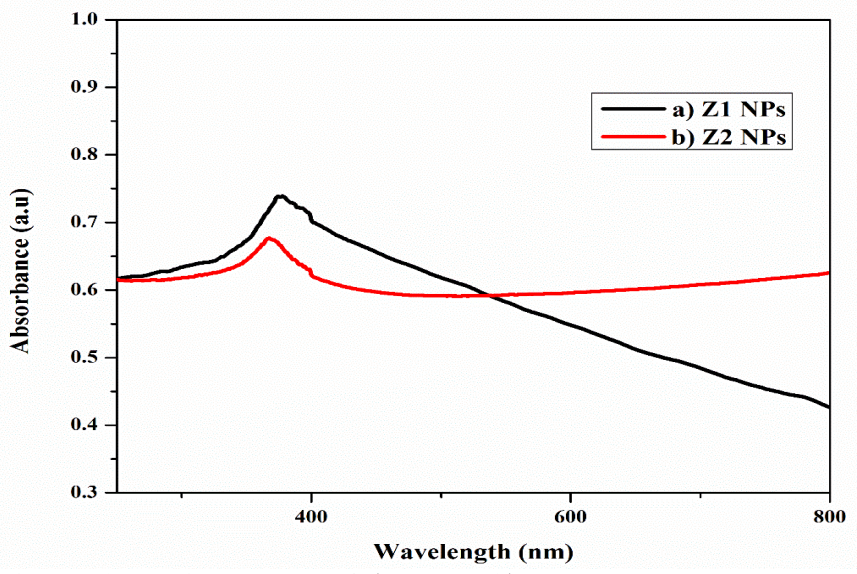

Fig. 5: Absorption spectra of a) Z1 NPs b) Z2 NPs

\section{Optical studies}

Fig. 5 shows that UV-visible optical absorption spectra of Z1 and Z2 NPs. From the absorption spectra, the absorption peaks are found at $377 \mathrm{~nm}$ for Z1 NPs whereas the peaks are observed at $366 \mathrm{~nm}$ for Z2 NPs, which can be attributed to the photo excitation of electrons from valence band to conduction band. It may due to the photo excitation of electrons from valence band to conduction band. [13]

Photoluminescence (PL) studies

Fig. $6(\mathrm{a}-\mathrm{b})$ show that the photoluminescence spectra of prepared Z1 and Z2 NPs. The PL emissions of very short wavelength of $350 \mathrm{~nm}$ to long wavelength $600 \mathrm{~nm}$ are observed for Z1 and Z2 NPs. The seven peaks for both Z1 and Z2 NPs are fitted by Gaussian function. The emission spectra have seven peak positions at 387 , 401, 416, 438, 457, 477, $516 \mathrm{~nm}$ and 357, 387, 411, 437, 450, 481, $497 \mathrm{~nm}$ for Z1 and Z2 NPs respectively. The observed PL bands are one violet emission, three blue emissions, one blue-green emission, two green emissions respectively.

The lowest wavelength of UV emission peaks are observed at 357 and $387 \mathrm{~nm}$, which correspond to the near-band emission (NBE) of Z1 and Z2 NPs. The four violet emissions centred at 410,416, 438 and $447 \mathrm{~nm}$ is attributed to an electron transition from a shallow donor level of the natural zinc interstitials to the top level of the valence band. [14] The blue green emission observed at 477, 481 and $497 \mathrm{~nm}$ is ascribed to the transition between the oxygen vacancy and interstitial oxygen. ${ }^{[15]}$ Finally two green emissions observed at 516 $\mathrm{nm}$, corresponds to the singly ionized oxygen vacancies. [16-17]
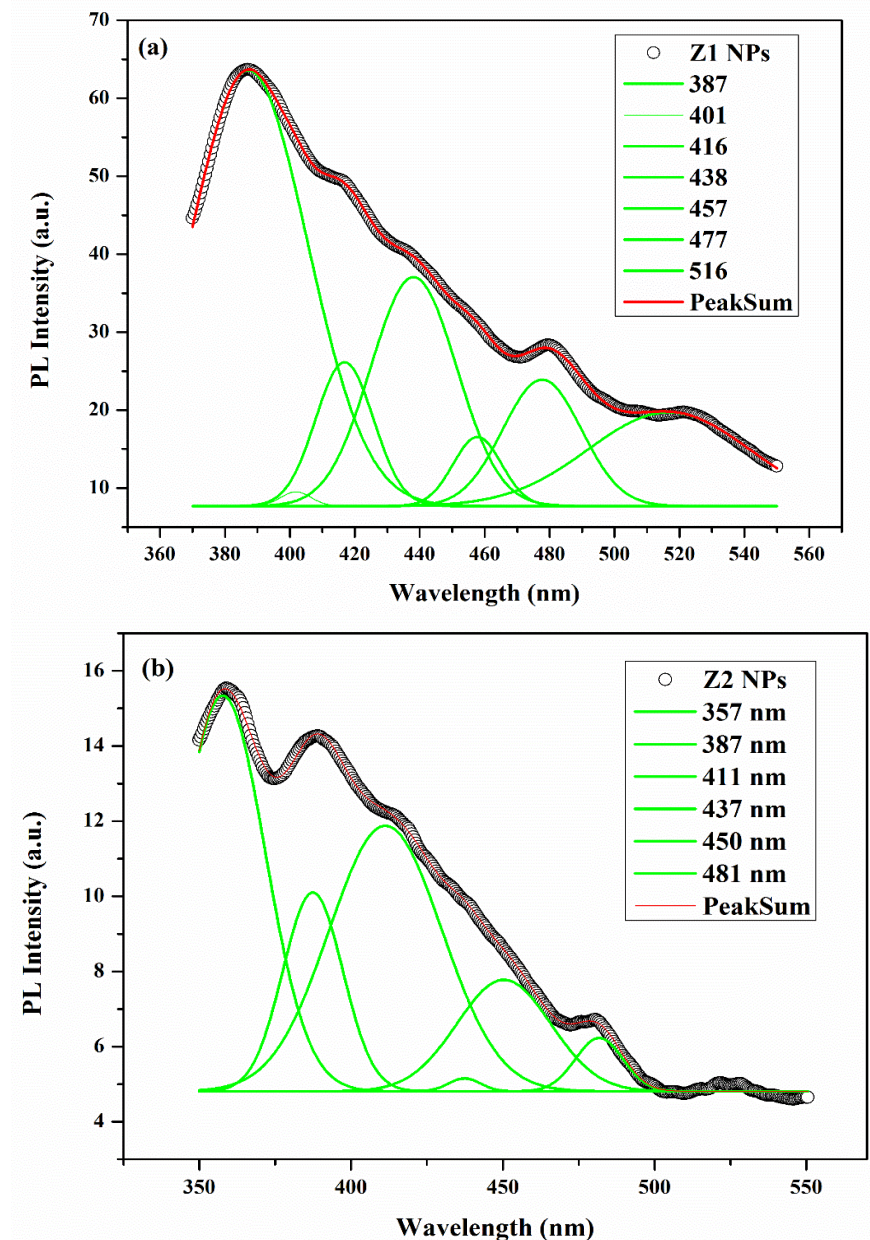

Fig. 6 (a \& b): photoluminescence emission spectra of Z1 NPs and Z2 NPs

Table 1: Antimicrobial activities of $\mathrm{Z1}$ and $\mathrm{Z} 2$ NPs for against human pathogens

\begin{tabular}{cccc}
\hline & & & \multicolumn{2}{c}{ Zone of inhibition $(\mathbf{m m})$} \\
\cline { 3 - 4 } S. & $\begin{array}{c}\text { Concentration of Z1 } \\
\text { No }\end{array}$ & $\begin{array}{c}\text { Gram positive } \\
\text { and } \mathbf{Z 2} \text { NPs }(\boldsymbol{\mu L})\end{array}$ & $\begin{array}{c}\text { Gram negative } \\
\text { strains }\end{array}$ \\
\cline { 3 - 4 } & & S. aureus & K. pneumoniae \\
\hline 1 & 15 & 1.7 & 1.4 \\
2 & 20 & 1.6 & 1.5 \\
3 & 25 & 1.5 & 1.3 \\
4 & 30 & 1.6 & 1.4 \\
5 & 35 & 1.7 & 1.4 \\
\hline
\end{tabular}

\section{Antimicrobial Activity}

In the present work, the antimicrobial activities of Z1 and Z2 NPs are shown in Fig.7. The antimicrobial activities of prepared Z1 and Z2 NPs were tested against the human pathogens like gram positive ( $S$. aureus) and gram negative (K. pneumoniae) bacteria with 
reference to Amoxicillin. The antimicrobial activity values are listed in Table 1. From the figure, the zones of inhibition values were increased with respect to the Z2 NPs concentration against the tested bacteria. The observed inhibition zone was higher in gram positive bacteria than gram negative bacteria which may be attributed to the difference in their cell wall structure of chemical composition. From these zones of inhibition values, it could be stated that Z2 NPs have higher antimicrobial property when compared to Z1 NPs. The antimicrobial activity of NPs may either directly interact with the microbial cells or produce secondary products that cause damage. ${ }^{[18]}$

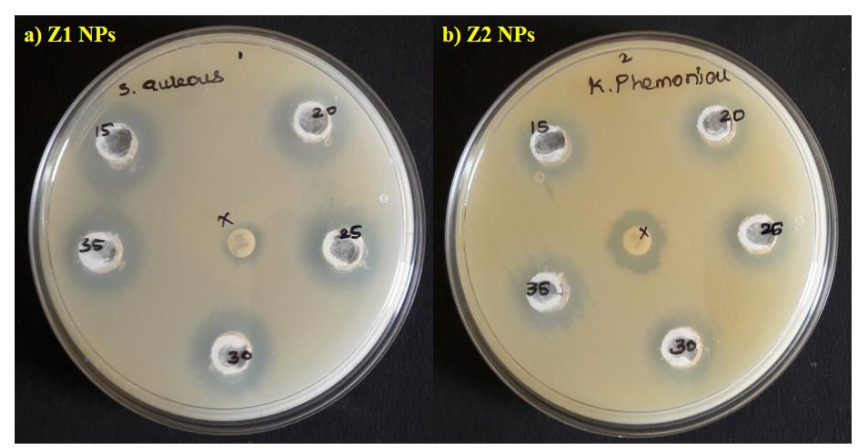

Fig. 7. Zone of inhibition of Z1 and Z2 NPs against S. aureus and $K$. pneumoniae

The Z1 and Z2 NPs were prepared successfully through co-precipitation method. The hexagonal wurtzite structure of Z1 and Z2 NPs were confirmed by X-ray diffraction studies. The calculated average crystallite sizes of $Z 1$ and $Z 2$ NPs were 42 and $35 \mathrm{~nm}$ respectively. The size of Z2 NPs is smaller when compared to Z1 NPs. This size reduction is mainly due to the distortion in the host $\mathrm{ZnO}$ lattice by the foreign impurities like $\mathrm{Sr}^{2+}$ and $\mathrm{Ba}^{2+}$ ions. From the FESEM images, the morphology of nanoparticles was found spherical and hexagonal in shape. The elemental compositions were identified for the synthesized Z1 and Z2 NPs using EDAX spectra. From the FT-IR spectra, the Zn-O stretching frequency appeared at $445 \mathrm{~cm}^{-1}$ and $523 \mathrm{~cm}^{-1}$ for Z1 and Z2 NPs. The UV-Vis spectrum showed the absorption peak is found at 377 and $396 \mathrm{~nm}$ for $\mathrm{Z} 1$ and Z2 NPs respectively. PL spectra showed that doping materials altered the band emission, which is due to zinc vacancy, oxygen vacancy and surface defects. The antimicrobial studies performed against a set of bacterial strains showed that the Z2 NPs possessed more antimicrobial property.

\section{ACKNOWLEDGMENT}

The authors are thankful to the members of Management committee and Principal of Jamal Mohamed College for providing necessary facilities.

\section{REFERENCES}

1. Look DC. Recent advances in $\mathrm{ZnO}$ materials and devices. Mater. Sci. Eng. B. 2001; 80: 383-387.

2. Ozgur U, Alivov YI, Liu C, Teke A, Reshchikov MA, Dogan S, Avrutin V, Cho SJ, Morkoc H. A comprehensive review of ZnO materials and devices. J. Appl. Phys. 2005; 98: 041301.

3. Ogale SB. Thin Films and Heterostructures for Oxide Electronics. Springer. New York, 2005.

4. Nickel NH, Terukov E. Zinc Oxide - A Material for Micro and Optoelectronic Applications. Springer. Netherlands, 2005.

5. Vandijken A, Meulenkamp EA, Vanmaekelbergh D. Meijerink A. The luminescence of nanocrystalline $\mathrm{ZnO}$ particles: the mechanism of the ultraviolet and visible emission. J. Luminesc. 2000; 87: 454-456.

6. Volbers N, Zhou H, Knies C, Pfisterer D, Sann J, Hofmann $\mathrm{DM}$, Meyer BK. Synthesis and characterization of $\mathrm{ZnO}: \mathrm{Co}^{2+}$ nanoparticles. Appl. Phys. A. 2007; 88: 153-155.

7. Nirmala M, Anukaliani A. Characterization of undoped and Co doped $\mathrm{ZnO}$ nanoparticles synthesized by DC thermal plasma method. Physica B. 2011; 406: 911- 915.

8. Maensiri S, Laokul P, Phokha S. A Simple Synthesis and Magnetic Behavior of Nanocrystalline $\mathrm{Zn}_{0.9} \mathrm{Co}_{0.1} \mathrm{O}$ Powders by Using Zn and Co Acetates and Polyvinyl Pyrrolidone as Precursors. J. Magn. Magn. Mater 2006; 305: 381-387.

9. Sankara Reddy B, Venkatramana Reddy S, Vijaya Lakshmi RP. Structural and optical properties of $\mathrm{Ag}$ and Co doped ZnO nanoparticles. AIP Conf. Proc. 2012; 1447: 431- 432.

10. Wright GD. Resisting resistance: New chemical strategies for battling superbugs. Cell Chem. Biol 2000; 7(6): 127-132

11. Zandi S, Kameli P, Salamati H, Ahmad H, Hakimi M. Microstructure and optical properties of $\mathrm{ZnO}$ nanoparticles prepared by a simple method. Physica B. 2011; 406: 32153218.

12. Sonawane YS, Kanade KG, Kale BB, Aiyer RC. Electrical and gas sensing properties of self-aligned copper-doped zinc oxide nanoparticles. Mater Res Bull. 2008; 43: 2719-2726.

13. Liu M, Kitai AH, Mascher P. Point defects and luminescence centres in zinc oxide and zinc oxide doped with manganese. J. Lumin. 1992; 54(1): 35-42.

14. Fan XM, Lian JS, Zhao L, Liu Y. Single violet luminescence emitted from $\mathrm{ZnO}$ films obtained by oxidation of $\mathrm{Zn}$ film on quartz glass. Appl Surf Sci. 2005; 252: 420-424.

15. Varghese N, Panchakarla LS, Hanapi M, Govindaraj A, Rao $\mathrm{CN}$ R. Solvothermal synthesis of nanorods of $\mathrm{ZnO}$, N-doped ZnO and CdO. Mater Res Bull. 2007; 42: 2117-2124.

16. Kumar N, Dorfman A, Hahm J. Fabrication of optically enhanced $\mathrm{ZnO}$ nanorods and microrods using novel biocatalysts. J. Nanosci. Nanotechnol. 2005; 5: 1915-1918.

17. Bagnall DM, Chen YF, Shen MY, Zhu Z, Goto T, Yao T. Room temperature excitonic stimulated emission from zinc oxide epilayers grown by plasma-assisted MBE. J Cryst Growth. 1998; 184: 605-609.

18. Karthikeyan M, Jafar Ahamed A, Vijayakumar P, Karthikeyan C. Green synthesis of pure $\mathrm{ZnO}$ and La doped $\mathrm{ZnO}$ nanoparticles and their structural, optical and antibacterial studies. Eur J Biomed Pharma Sci. 2018; 5: 736741.

HOW TO CITE THIS ARTICLE: Karthikeyan M, Jafar Ahamed A, Vijaya Kumar P. Pure and SrBa Dual Doped ZnO Nanoparticles Prepared by Co-precipitation Method and Their Structural, Optical and Antimicrobial Properties. Int. J. Pharm. Sci. Drug Res. 2019; 11(6): 284-288. DOI: 10.25004/IJPSDR.2019.110602 